\title{
Air Switch Remote-control System Based on GSM network
}

\author{
Zou En ${ }^{1,2}$, Huo Qing ${ }^{2}$, Huang Shuihong ${ }^{2}$,Chen Shengchuang ${ }^{1}$ \\ (1. College of zhujiang. South China Agricultural University. Guangzhou 510900, P.R.China. \\ 2.College of Engineer. South China Agricultural University. Guangzhou 510642, P.R.China.
}

Keywords: air switch; GSM network; remote control; STC12C5052AD single-chip microcomputer;

\begin{abstract}
TO solve the problem of air switch cannot be controlled remotely, an air remote control switch system is designed based on GSM network. Specific content information issued by the emission module of mobile terminal of system and received by GSM module, then decoded by the STC12C5052AD to make a real-time control of the relay achieving remote control of air switch. Specific content information is issued by the emission module of mobile terminal of system and received by GSM module. Time and place is not be limited in the system, real-time trip and timing trip can also be achieved, and could close manually only be assigned in specified period.
\end{abstract}

At present, both in the industrial electricity or household electricity, air switch plays an important protective role, but do not have the timing of the air switch tripping and remote tripping of function, not from artificial close control. If you forget the staff after work off electrical power to the device must be able to have access to the power switch to turn off the power of man; and if need remote emergency shutdown of all electrical equipment in the building, or in the timing off after work, let those present to consciously leave, which require manual operation ${ }^{[1]}$ 。

Remote control switches on the market today are mostly used on short-distance transport in the form of electromagnetic waves or Ethernet, the disadvantage is the short distance, the remote control device for power is limited, single function. With the continuous development of global mobile communication technology, SMS GSM wireless technology (SMS) online at any time because it has a wide range of coverage, high reliability, has been widely used in a variety of data communication equipment ${ }^{[2]}$.

This paper presents a free from time and place, the use of mobile remote achieve instantaneous trip, specify the time closing air switch control system, the switch-off time can be modified in real time via the phone keypad. At present, the system has passed the user acceptance, in construction applications can be easily standardized management of electricity.

\section{Control System Design}

Remote control switch system includes a mobile terminal air emission module, GSM receiver module, the main chip STC12C5052AD, relay module, air switch module, clock module. First set the only way to control the device's phone number, the phone number by the end of the transmitter module to issue a set of identifiable information, air switch can control the timing and ready to trip in the microcontroller program. Every air switching the system to send the air switch status information to mobile phones, mobile phone users can obtain real-time feedback to understand the system is working properly. After the number of SIM card SMS reaches 50, the microcontroller sends clear message command to ensure the normal operation of GSM module to ensure stable operation of the system for a long time.

PCF8563 clock module provides the clock signal and GSM module receives information on obtaining the mobile network end the microcontroller standard time, the time clock chip calibration to ensure the accuracy of time; the time set by the master chip to do with the time clock module compares send control command, and the set time command modified at any time by mobile phone signals, each state modified time and air switch is stored in EEPROM microcontroller comes in to ensure the system after previous air power to maintain the state of the switch, meet timing control air switch requirements. System control block diagram shown in Figure 1. 


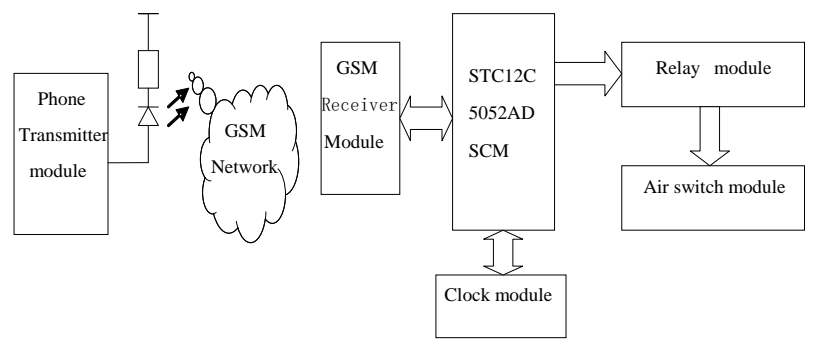

Fig.1 Diagram of control system

\section{System hardware design.}

Hardware parts include: Part 5 MCU MCU module ${ }^{[3]}$, GSM module, clock module, relay module, air switch module.

\subsection{Relay Module}

Relay is an electronic control device that can be used to control a smaller current large current "automatic switch", it plays the circuit automatically adjusts the role of security, conversion circuit. Relay module SRD-5VDC-SL-C loose music relay, operating voltage DC5V, can control the AC220V electrical equipment for controlling air switch trip.

\subsection{Air switch module}

Selection Chint air switch DZ47LE, the switch uses VG54123 chips form leakage protection with leakage, overcurrent protection functions, air switch internal circuit shown in Figure 2.

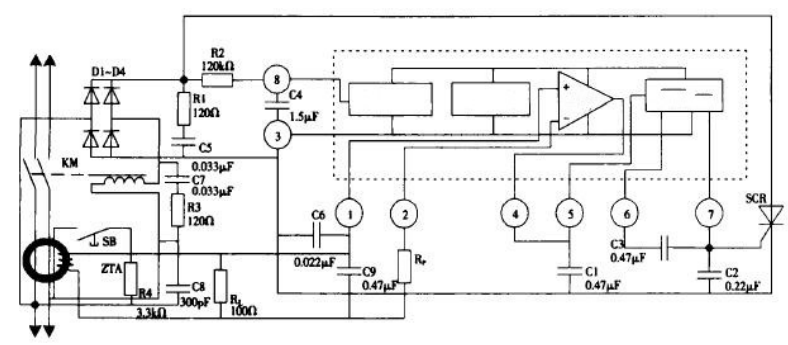

Fig.2 Circuit diagram of air automatic switch

Rectifier bridge D1-D4; KM relay; SB is normally open button, ZTA zero sequence current transformer, monitoring the leakage current; SCR thyristors, within the dashed box VG54123 internal circuit diagram of the chip. SB When the button is pressed, the air switch automatically trip. Based on this principle, the relay output circuit in parallel with the SB button, relay closed to control air switch trip, the formation of a new remote-controlled switch.

\section{Software Design}

C51 language is used programming in Keil uVision4 environment commissioning. Program includes hardware initialization process, GSM module program ${ }^{[4]}$,PCF8563 clock chip and interrupt service routine. GSM module receives the phone sends the message and echoed back to MCU; clock chip program is to provide a clock signal to the MCU, the MCU processing clock signal to achieve regular trip; interrupt service routines include intercept and handle phone signal content.

\subsection{Programming}

\subsubsection{The main design}

The main program flow chart is shown in Figure 3. MCU initialization, including serial ports, timers, IO ports, etc. initialization; GSM module initialization, including short messaging control mode is set to Text mode, set the short message service center is set to Guangdong Mobile and serial echo mode; wait 30 seconds, after a successful search GSM network system into normal operation began waiting for an interrupt. In addition, the clock chip provides a clock signal, the system is time detection state. If the set trip time is 17:00, the air switch automatically trip, if set to 
allow the closing time of 7:00 am, at the moment to allow the closing, and the online system returns to SMS mobile phone users to avoid network signal is not good cause because the system did not perform an action but the user does not know. Interrupt arrives, execute the interrupt routine, or time has been detected.

\subsubsection{Interrupt subroutine design}

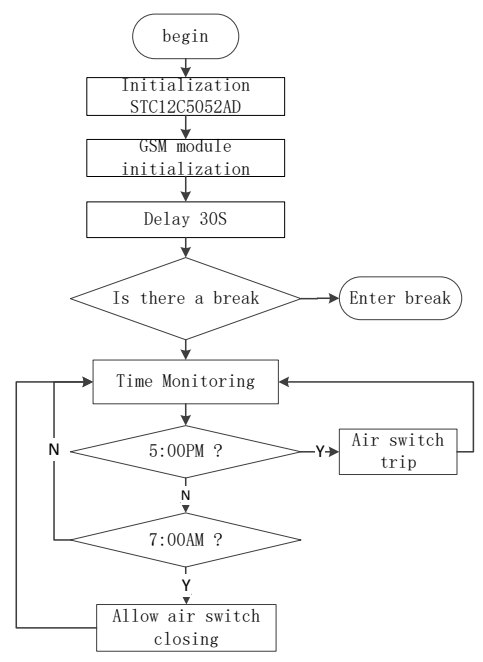

Fig.3 Flow chart of master program

The main content of the interrupt subroutine is the phone number to receive specific information and the content information extraction facilitates single-chip processing, to meet the various mobile phone command operations. Interrupt subroutine flow chart shown in Figure 8, first determine whether it has received a specific phone number to call, then GSM module echo the content of messages to the microcontroller, the microcontroller and the serial number of the interception of messages which sends a read command, such as reading the content "open", the MCU P3.5 port set; such as reading the content of "setopentime: 17: 00", then set air switch tripping at 17:00.

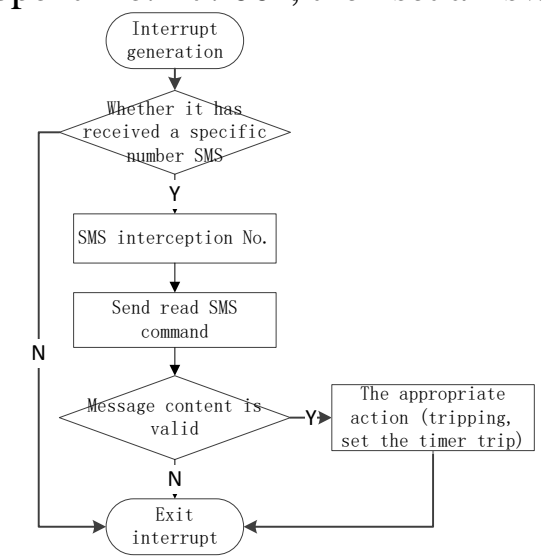

Fig.4 Flow chart of interrupt function

\subsection{Wireless module TC35I software analysis}

Decoding and encoding text messages are the core software programming. AT modem command set is an industry standard communication interface, the modem is to identify and execute orders. Through serial communication, the microcontroller can send a series of AT commands to control the module to module. Send commands and instructions received are ASCII character codes in microcontroller programming should pay attention to string conversion.

When the program starts, we need to send commands to initialize TC35I, where "AT + CNMI = $1,1,2 \backslash \mathrm{r} \backslash \mathrm{n}$ " is used to set the short message arrives when automatic echo; "AT + CMGF $=1 \backslash \mathrm{r} \backslash \mathrm{n}$ ", set text encoding mode, in text mode, sending and receiving information is in text format; "AT + CSCA $=+8613800200500 \backslash \mathrm{r} \backslash \mathrm{n}$ " set the short message service center address; 3 instructions after 
sending the GSM initialized.

\section{Conclusion}

This paper set up a remote control air switch system based on GSM network, without the constraints can be realized at any time and timing the trip, and the trip time to modify the transmitted signal through mobile terminals, can facilitate power management. System uses existing GSM wireless network, the signal can cover a radius of maximum $35 \mathrm{~km}$, to overcome the traditional short distance wireless switch control, control restrictions and other electrical power disadvantages and get rid of the traditional manual control switch only to increase the timing of the trip function. In addition, the design can also be used Dormitory power management and smart home remote management.

\section{Fund Project}

Guangdong Province, province, the higher education innovation and strong school project (4724) project funding.

\section{Reference}

[1]Zhoumi. The development direction of the air circuit breaker and product development [z], Electric Age, 2006 (1): 11-13

[2]Zhang Huadong, Qinjian Chun, design and development of mobile ground Liu remote online monitoring system [J] Automation and Instrumentation, 2007 (2): 45-48

[3]Wang Jing Science and Technology. STC12C5052AD MCU Device Handbook [z], 2009.

[4]Dong Dianyong, Jiang Ping wireless micro printer based on GSM short message [J] Automation and Instrumentation, 2013 (2): 33-36 